\title{
The impact of scheduling on service reliability: trip-time determination and holding points in long-headway services
}

\author{
N. van Oort $\cdot$ J.W. Boterman $\cdot$ R. van Nes
}

Published online: 12 June 2012

(C) The Author(s) 2012. This article is published with open access at Springerlink.com

\begin{abstract}
This paper presents research on optimizing service reliability of longheadway services in urban public transport. Setting the driving time, and thus the departure time at stops, is an important decision when optimizing reliability in urban public transport. The choice of the percentile out of historical data determines the probability of being late or early, while the scheduled departure time determines the arrival pattern for travelers. A hypothetical line and a case study are used to determine the optimal percentile value for long-headway services without and with holding points. If no holding points are applied, it is shown that the 35-percentile value minimizes the additional travel time to $25 \%$ of the reference situation. In the case of holding, two holding points combined with a 30-60-percentile value yield the best performance: a further reduction of the additional travel time with $60 \%$.
\end{abstract}

Keywords Urban public transport · Service reliability · Timetable design · Holding

\section{Introduction}

The quality of public transport is determined by many aspects, such as availability, comfort, travel time and costs. Reliability of travel time has become increasingly important over the last decade. Deviations of the timetable result in additional travel

\footnotetext{
N. van Oort $(\bowtie)$

Goudappel Coffeng Mobility Consultants, P.O. Box 16770, 2500 BT The Hague, The Netherlands e-mail: NvOort@Goudappel.nl

N. van Oort · R. van Nes

Delft University of Technology, Faculty of Civil Engineering and Geosciences, Transport \& Planning, P.O. Box 5048, 2600 GA Delft, The Netherlands 
Fig. 1 (Improving) reliability

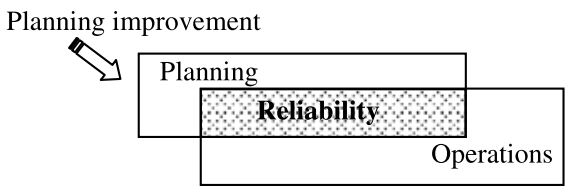

Operations improvement

time and reduce the probability of finding a seat (Van Oort 2011). In addition, service reliability strongly influences mode choice, as stated, for instance, in Van Oort and van Nes (2009b). In urban public transport, service reliability is not optimal. A case study in The Hague in The Netherlands shows the effects of unreliability on travel time of passengers. The analysis of actual data of buses and trams shows that travel time can be extended by $25 \%$ due to distribution in trip times. Van Oort (2011) states that the focus of research and applications of improvements in reliability is often on the operational level: the network and timetable are given and the only way to improve reliability is by adjusting operations such that they are better aligned with the timetable (e.g. as shown in Chang et al. 2003; Chowdhury and Chien 2001; Furth and Muller 2009; Levinson 1991; Muller and Furth 2000 and Wilson et al. 1992).

Figure 1 illustrates how reliability is defined: the match of planning and operations.

To achieve the highest level of reliability, planning and operations should be equal. Improving service reliability is thus a matter of adjusting one of these aspects to the other. The hypothesis in Van Oort (2011) is that by both adjusting operations and planning, opportunities are created to improve the match. This paper focuses on optimizing timetables for long-headway services, both from a theoretical and a practical perspective, the latter by conducting a case study in the city of The Hague. Variation of operations is known and the timetable is adjusted to create an optimal match, i.e. achieving minimal travel time for passengers. Figure 2 shows the elements of the travel time.

This research focuses on the part of the journey from the arrival of the passenger at the origin stop until the arrival of the passenger at the destination stop. Transfers are not included. This part of the journey consists of waiting time at the origin stop and the time spent in the vehicle. It is assumed that travellers use the schedule to determine their arrival time at the stop. The waiting time thus depends on the difference between the scheduled and the actual departure of the vehicle: In other words, the level of schedule adherence determines the amount of additional time the passenger has to wait compared to the situation where all vehicles operate perfectly on time. In-vehicle time and its variation are assumed to be constant. A typical measure to improve punctuality is to apply a holding strategy. In that case, the waiting time of vehicles (and passengers) due to holding should also be taken into account.

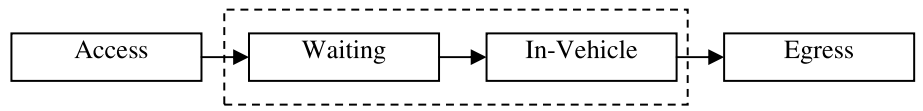

Fig. 2 Elements of travel time and research focus 
The paper is organised as follows: Sect. 2 explains the design process of the timetable and its main variable, namely trip times (both stop to stop and terminal to terminal). The application of holding points is also explained. A calculation model is described in Sect. 3, enabling calculation of the additional travel time as a function of choices in the timetable design process. A theoretical analysis is made of a hypothetical line as well as a case study, using actual data of trip time and passenger flows in the city of The Hague. The analysis first deals with the case without holding strategies and then with the use of holding points. The results of both analyses are shown in Sect. 4. The paper ends with conclusions.

\section{Timetable design}

This paper focuses on the tactical stage of urban public transport design. During this phase, the timetable is constructed. A variety of factors are important when designing a proper timetable. Given a public transport network, frequencies should be determined: how many departure possibilities are provided per hour? This relates to both quality and capacity; how many seats are offered per hour, and is a sufficient level of comfort achieved? Coordination with other lines is important as well during the timetable design: this applies to transfer options and to offering a constant interval on a track with more than one line. Through this coordination, the departure times in the timetable are fixed exactly on time. However, the key input in this process are the scheduled driving times of each line. This is therefore the topic this paper focuses on.

\subsection{Trip time}

The main element of a timetable, besides the number of runs, is the trip time, i.e. the time necessary to drive from stop to stop and finally from terminal to terminal. In urban public transport, unlike heavy rail, it is common practice to determine the trip time based on empirical data from the previous period. Using this type of feedback, attainable trip times are scheduled. An example of empirical data of trip times is illustrated in Fig. 3: the realized trip times of tram line 1 in The Hague (ScheveningenDelft, working days, morning peak, April 2007).

Figure 3 shows that the realized trip times have a wide distribution. This is caused by several factors, such as weather, traffic, variation in the number of passengers, human interaction, etc. Cham and Wilson (2006) provide a detailed description of possible sources. The challenge for the planner is to choose the best trip time out of this distribution, since only one time can be used as input for the timetable. By choosing a single trip time, while knowing the real trip times are distributed, schedule adherence will not be optimal: without further measures, differences will arise between the timetable and actual operations.

The trip time is normally chosen by selecting a percentile value (by over $75 \%$ of the operators as shown by an international survey (Van Oort 2009)). Figure 3 indicates the 15-, 50- and 85-percentile values. It is common use in urban public transport to select a high percentile value, achieving a high level of attainability.

Muller and Knoppers (2004) presented research, recommending the 85-percentile value, which leads to an attainable timetable. The first analysis will explore what the 


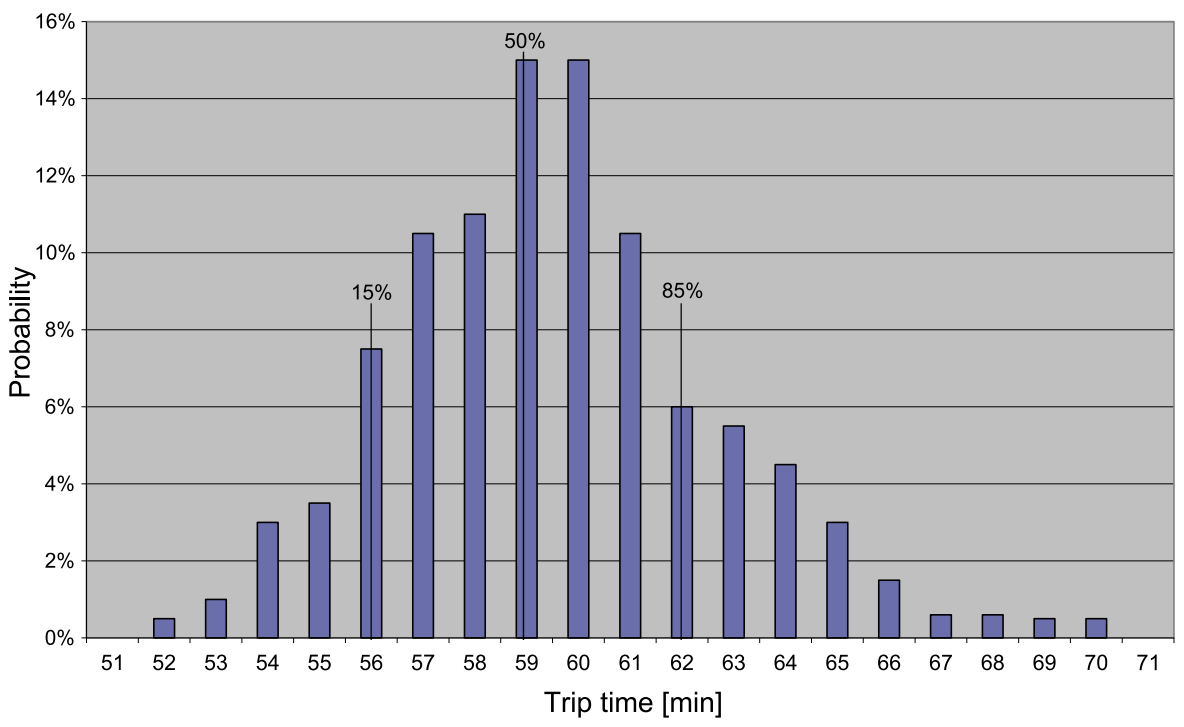

Fig. 3 Distribution of trip times for tram line 1 and percentile values
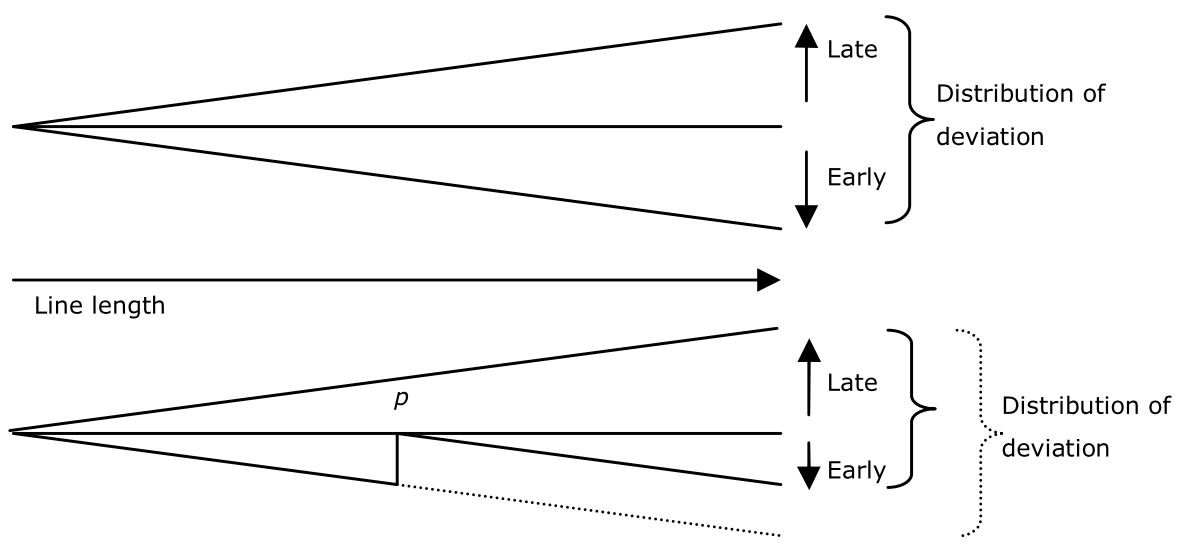

Fig. 4 Effect of holding point $p$ on schedule adherence

optimal percentile value is in case of the objective of minimizing additional travel time.

\subsection{Holding points}

Holding points are stops where drivers are not allowed to leave ahead of schedule. Introducing holding points will affect service reliability and travel time. Not driving before the scheduled time reduces the variation in trip times and the distribution of schedule deviation, as Fig. 4 shows. At holding point $p$, vehicles that have arrived early wait until they are on time. This decreases the width of the distribution of the 
deviation. As described by Van Oort and van Nes (2009a), holding points are used, for example, at RandstadRail (light rail service in The Hague).

In this paper, we present the results of a research study on designing trip time and holding points: how many points should be introduced and what percentile value should be used for the driving towards the holding point to minimize additional travel time.

Note that not every stop can be used as a holding point. At a good holding point, the proportion of travellers who board and alight is high. People who travel from a stop before the holding point to a stop after, gain no advantage from waiting at the holding point. In the same way as used in trip time determination, the trip time from holding point to holding point can also be chosen by a percentile value out of a distribution of realized trip times.

\subsection{Consequences of trip time determination and holding points}

The final choice of the trip times has many consequences. Both implying the supply and demand sides, the main ones are:

- The probability of departing on time at the first stop

If many vehicles are delayed, the probability of a punctual departure of the next run will decrease. This depends both on the delay and on the slack in the layover time at the terminus.

- Attainability for the driver

The longer the scheduled trip time, the larger the probability that a driver will arrive within the scheduled time. The consequence could be that drivers are ahead of schedule.

- Number of vehicles needed

The number of vehicles required to operate the timetable is determined by the frequency and cycle time of the line. This time consists of trip time and layover time in two directions. If the scheduled trip time decreases, one vehicle is able to run more runs, and fewer vehicles are required. However, no fractions of vehicles can be used, so a reduction will not automatically decrease the number of vehicles. If the scheduled trip time is not attainable, the frequency will drop and/or the actual layover will decrease.

- Travel time of passengers

As illustrated by Fig. 2, the total journey does not only consist of trip time. Waiting comprises a substantial part of the trip as well, especially in urban transport. If operations do not match the planning, the waiting time at the stop increases (Van Oort and van Nes 2008). If vehicles drive ahead of schedule, it is possible that passengers miss their vehicle and have to wait a complete headway. The choice of which percentile value of the historical data is used for a new timetable determines the punctuality and therefore waiting time and travel time.

The choice of the optimal percentile value is a major topic in many public transport companies (as shown by an international survey (Van Oort 2009)). The effects mentioned above show the large consequences of the choice of scheduled trip time. This paper describes a quantitative analysis of the choice of percentile value and application of holding points. Two effects are shown: the additional travel time caused 
by the mismatch of planning and operation and the probability of departing on time at the first stop for the next run. In analyzing the effect of trip times, the following aspects are of primary interest:

- The distribution of trip times: the larger the variability, the larger the effect on punctuality and probability to arrive at the last stop on time.

- Frequency: The smaller the frequency, the larger the effect of driving ahead of schedule. After all, people have to wait a complete headway if they miss the vehicle.

- Number of boardings. The stops where people board and their location along the line are of great importance.

- Layover time: The time at the last stop, before departing in the other direction can be used as slack time to make up for delays. A portion of this time can also be used as a break for the drivers. This portion is not taken into account in this research.

The distribution of actual trip times is assumed to be fixed in this research; only the scheduled trip time is changed. Concerning further research, it is important to consider the interaction between the scheduled and actual trip time. More information will become available for the driver, affecting his driving style (Carey 1998).

The scheduled time is communicated to passengers and it is assumed that they adjust their moment of arrival at the departure stop according to the scheduled departure time, which determines their waiting time. As mentioned earlier, the effects of driving ahead of schedule are considerable, i.e. an extension of waiting time by a complete headway. In the second analysis, the research results are presented of the strategy where vehicles are not allowed to depart ahead of schedule: the introduction of holding points. In this case, the number of passengers in the vehicle is used as additional input. Due to the fact that vehicles are not allowed to depart early, these passengers could have to wait at the stop. This leads to additional travel time for the passing passengers.

\section{Calculation model}

A model is designed to analyze the effect of the choice of trip time and holding points on punctuality, travel time and the probability of on-time departure. This section describes this model and the algorithms used, as well as specifying the data used.

\subsection{Calculation of punctuality and additional travel time}

The first step in the model is constructing the timetable. The main variable is the percentile value, which is used to determine the scheduled trip time based on the data of actual trip times during the previous period. Once the percentile value is chosen, the complete timetable can be determined. The main input is, of course, actual data from the operations, i.e. trip times from stop to stop on a certain line. The next step is to compare the newly constructed timetable with the stochastic set of trip times. This is under the assumption that trip times are fixed, i.e. not affected by the timetable. 
Now the deviation of the timetable can be calculated, using formula (1) (Hansen 1999).

$$
p_{l}=\frac{\sum_{j}^{n_{l, j}} \sum_{i}^{n_{l, i}}\left|\tilde{D}_{l, i, j}^{a c t}-D_{l, i, j}^{s c h e d}\right|}{n_{l, j} \cdot n_{l, i}}
$$

where:

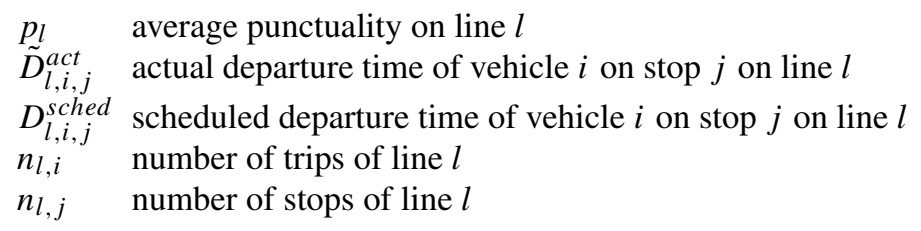

Punctuality is a commonly used indicator for service reliability, but does not take into account the difference between the effect of driving ahead of schedule or driving late and does not incorporate the impact of passenger patterns along the line. To consider this, a new indicator is introduced, namely the average additional waiting time per traveller due to variety of trip times (Van Oort 2011).

When calculating the average additional travel time per passenger, two situations have to be distinguished, namely planned or random arrivals of passengers at the stop. If passengers arrive at random, exact departure times are not relevant anymore. In this paper, the focus is only on planned arrivals matching with long-headway services. Van Oort et al. (2010) elaborate on random arrivals and the effects on additional travel time. Main assumptions in the calculation model (Van Oort 2011) are:

- The examined period is homogeneous concerning scheduled departure times, trip times and headways (for instance off peak hour on working days in a month);

- The passenger pattern on the line is assumed to be fixed (in the analyzed period);

- All passengers are able to board to the first arriving vehicle.

Equations (2)-(4) show the computation of the average additional travel time per passenger (Van Oort 2011). Passengers are assumed to arrive randomly between the scheduled departure time minus $\tau_{\text {early }}$ and plus $\tau_{\text {late }}$ and it is assumed that in that case they do not experience any additional waiting time if the vehicle departs within this time window. Van Oort (2011) presents empirical research of these values. It is important to note that there is a difference between driving ahead of schedule and driving late. Driving ahead (i.e. departing before the scheduled departure time minus $\tau_{\text {early }}$ ) leads to a waiting time equal to the headway ( $H_{l}^{\text {sched }}$; assuming punctual departure of the successive vehicle). Especially in the case of low frequencies, this leads to a substantial increase in passenger waiting time. Driving late creates an additional waiting time equal to the delay $\left(\tilde{d}_{l, i, j}^{\text {departure }}\right)$.

The additional waiting time is first calculated per stop (Eqs. (2) and (3)) and next it is computed as a weighted average for all passengers on the line (Eq. (4)), depending on the number of boardings per stops.

$$
\begin{cases}\tilde{T}_{l, i, j}^{\text {Add,waiting }}=H_{l}^{\text {sched }} & \text { if } \tilde{d}_{l, i, j}^{\text {departure }} \leq-\tau_{\text {early }} \\ \tilde{T}_{l, i, j}^{\text {Add,waiting }}=0 & \text { if }-\tau_{\text {early }}<\tilde{d}_{l, i, j}^{\text {departure }}<\tau_{\text {late }} \\ \tilde{T}_{l, i, j}^{\text {Add,waiting }}=\tilde{d}_{l, i, j}^{\text {departure }} & \text { if } \tilde{d}_{l, i, j}^{\text {departure }} \geq \tau_{\text {late }}\end{cases}
$$




$$
\begin{aligned}
E\left(\tilde{T}_{l, j}^{\text {Add,waiting }}\right) & =\frac{\sum_{i} E\left(\tilde{T}_{l, i, j}^{\text {Add, waiting }}\right)}{n_{l, i}} \\
E\left(\tilde{T}_{l}^{\text {Add,waiting }}\right) & =\sum_{j}\left(\alpha_{l, j} \cdot E\left(\tilde{T}_{l, j}^{\text {Add,waiting }}\right)\right) \quad \text { with } \sum_{j} \alpha_{l, j}=1
\end{aligned}
$$

where:

$E\left(\tilde{T}_{l, i, j}^{\text {Add,waiting }}\right)$ average additional waiting time per passenger due to unreliability $H_{l}^{\text {sched }}$ of vehicle $i$ of line $l$ at stop $j$

$\tilde{d}_{l, i, j}^{\text {departure }}$

$\tau_{\text {early }}$ departure deviation of vehicle $i$ at stop $j$ on line $l$

$\tau_{\text {late }}$ lower bound of arrival bandwidth of passengers at departure stop

$n_{l, i}$ upper bound of arrival bandwidth of passengers at departure stop

$\alpha_{l, j}$ number of trips on line $l$ proportion of passengers of line $l$ boarding at stop $j$

When holding points are used, passenger waiting time in the vehicle must be considered in addition to passenger waiting time at the stop. Equations (5)-(10) show the computation of the impact of holding on waiting in the vehicle, including the calculation of the average effects for all passengers (Eq. (10)). Note that due to holding, the additional waiting time at the stop downstream of the holding point decreases, due to enhanced schedule adherence.

$$
\begin{aligned}
& \tilde{T}_{l, i, j}^{\text {holding }}=D_{l, i, j}^{\text {sched }}-\tilde{D}_{l, i, j}^{a c t} \quad \text { if } j=h_{n} \text { and } \tilde{D}_{l, i, j}^{a c t}<D_{l, i, j}^{s c h e d} \\
& \tilde{T}_{l, i, j}^{\text {holding }}=0 \quad \text { if } j=h_{n} \text { and } \tilde{D}_{l, i, j}^{a c t}>D_{l, i, j}^{s c h e d} \\
& \tilde{T}_{l, i, j}^{\text {holding }}=0 \\
& \text { if } j \neq h_{n} \\
& \tilde{T}_{l, i, j}^{a d d, \text { in-vehicle }}=\tilde{T}_{l, i, j}^{\text {holding }} \\
& \tilde{D}_{l, i, j}^{\prime a c t}=\tilde{D}_{l, i, j}^{a c t}+\sum_{1}^{j} \tilde{T}_{l, i, j}^{\text {holding }} \quad j \geq h_{1} \\
& T_{l, j}^{a d d, \text { in-vehicle }}=\frac{\sum_{i=1}^{n_{l, i}} T_{l, i, j}^{a d d, \text { in-vehicle }}}{n_{l, i}} \\
& T_{l}^{a d d, \text { in-vehicle }}=\sum_{j=1}^{n_{l, j}} \beta_{l, j} \cdot T_{l, j}^{a d d, \text { in-vehicle }} \\
& T_{l}^{a d d}=T_{l}^{a d d, \text { waiting }}+T_{l}^{\text {add,in-vehicle }}
\end{aligned}
$$

where: 
$T_{l, i, j}^{a d d, \text { in-vehicle }}$ expected additional in-vehicle time in vehicle $i$ at stop $j$ on line $l$

$T_{l, i, j}^{a d d, \text { waiting }} \quad$ expected additional waiting time due to vehicle $i$ at stop $j$ on line $l$

$T_{l}^{a d d} \quad$ average additional waiting time per passenger on line $l$

$\beta_{l, j} \quad$ proportion of passengers passing stop $j$ on line $l$

\subsection{Calculation of probability of departing on time}

In addition to the average additional travel time per passenger, the probability of on-time departure at the first stop is determined by the model $\left(P_{l, i, 1}^{\text {on } t \text { time-departure }}\right)$. The model calculates the punctuality deviation at the last stop for every vehicle trip $\left(\tilde{d}_{l, i, \text { last_stop }}^{\text {arrival }}\right)$ and uses this value in combination with the scheduled layover time (i.e. the time between the scheduled arrival and departure of the vehicle at the terminal, $T_{l, i}^{\text {layover }}$ ) to determine the departure delay (if the arrival delay is larger than the layover time; otherwise, no delay will occur). After the departure delay for all vehicle trips has been calculated, the probability of on-time departure is determined. Equation (11) shows the calculation of the probability of departing on time.

$$
P_{l, i, 1}^{\text {on_time-departure }}=P\left(T_{l, i}^{\text {layover }}-\tilde{d}_{l, i, \text { last_stop }}^{\text {arrival }} \geq 0\right)
$$

where:

$P_{l, i, \overline{1}}^{\text {on_time-departure }}$ probability of on-time departure of vehicle $i$ at the terminal on

$T_{l, i}^{\text {layover }}$ line $l$

$\tilde{d}_{l, i, \text { last_stop }}^{\text {arrival }}$ scheduled layover time of vehicle $i$ at the terminal on line $l$ arrival deviation of vehicle $i$ on line $l$ at the terminal

\section{Results}

The study on minimizing the average additional travel time per passenger by adjusting the timetable is set as follows. It consists of both a case study of actual lines in The Hague and an analysis of a hypothetical line. In this way, the analysis yields practical valuable results and by adding the second analysis, the variables are controllable so effects of different values may be calculated.

The input for the theoretical analysis is a fictitious line with a trip time of 30 minutes, headways of 15 minutes, serving 30 stops. The boardings and alightings are distributed across the line, as Fig. 5 shows. This service line is assumed to be a typical radial line, in which boardings mainly occur in the first part of the line and alightings in the second part.

Based on actual trip time data, the trip times are assumed to be Gaussian distributed (as proposed by Abkowitz et al. 1987 and Strathman et al. 2002). Three scenarios are analyzed, namely actual trip times with $5 \%, 10 \%$ and $20 \%$ of the scheduled trip time as standard deviation. Using these values, a set of hypothetical "actual" trip times is randomly determined which is the input for the analysis, as described in Sects. 4.1.1 and 4.2.1.

The case study consists of different tram and bus lines (in terms of length and distribution of trip times) operated by HTM in The Hague. Actual trip times and 


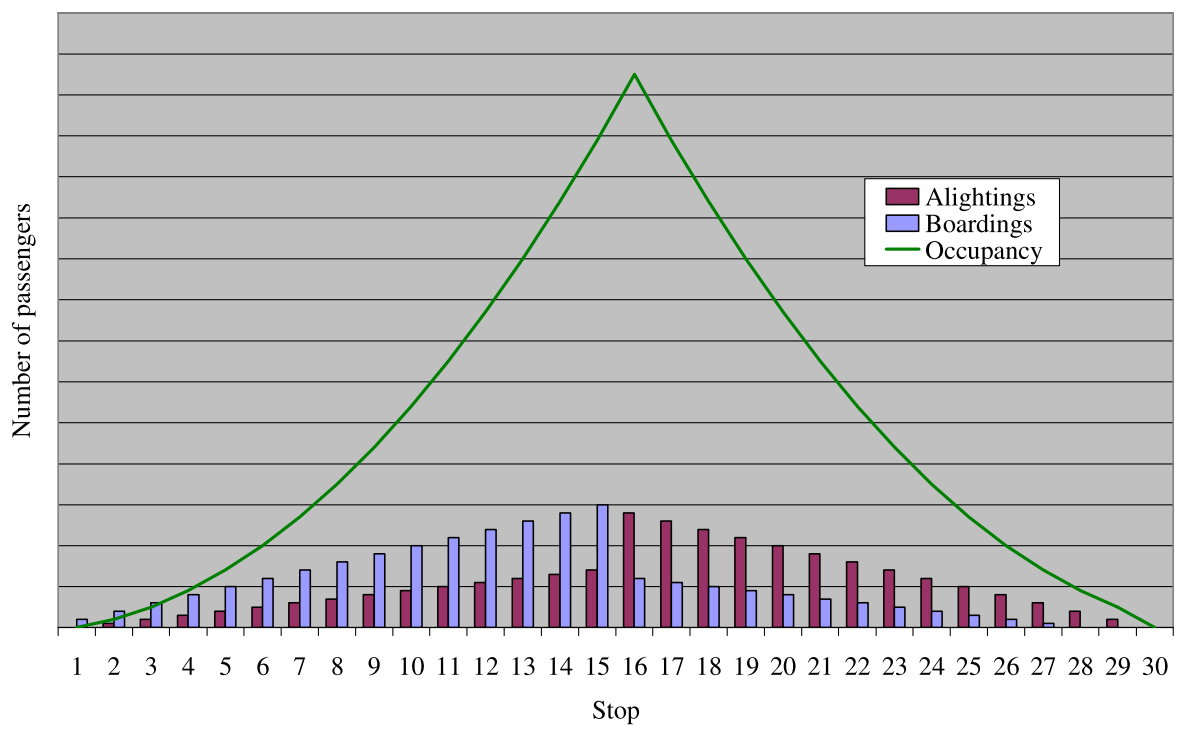

Fig. 5 Distribution of boarding and alighting for the hypothetical $l$ line

Table 1 Characteristics tram lines case study ${ }^{\mathrm{d}}$

\begin{tabular}{lllllll}
\hline Line & From & To & $\begin{array}{l}\text { Length } \\
{[\mathrm{km}]}\end{array}$ & $\begin{array}{l}\text { Scheduled } \\
\text { trip time } \\
{[\mathrm{min}]}\end{array}$ & $\begin{array}{l}\text { Standard } \\
\text { deviation } \\
{[\mathrm{min}]}\end{array}$ & $\begin{array}{l}\text { Relative } \\
\text { Standard } \\
\text { deviation }\end{array}$ \\
\hline $1^{\mathrm{a}, \mathrm{b}}$ & Scheveningen & Delft & 20.0 & 60 & 3 & $5 \%$ \\
$2^{\mathrm{b}}$ & Kraayenstein & Leidschendam & 13.0 & 43 & 3 & $6 \%$ \\
$9^{\mathrm{a}}$ & Vrederust & Scheveningen & 13.7 & 40 & 4 & $10 \%$ \\
$11^{\mathrm{a}}$ & Scheveningen & Den Haag HS & 8.1 & 22 & 2 & $9 \%$ \\
$15^{\mathrm{b}}$ & Moerwijk & Nootdorp & 16.7 & 52 & 3 & $7 \%$ \\
$17^{\mathrm{a}}$ & Statenkwartier & Wateringen & 16.4 & 42 & 3 & $7 \%$ \\
$18^{\mathrm{b}}$ & Clingendael & Rijswijk & 14.5 & 49 & 7 & $15 \%$ \\
\hline
\end{tabular}

a Working days, April 2007

b Working days, February and March 2007

c Percentage of trip time

d At http://www.htm.net/smartsite.dws?id=169 a network map is available

actual passenger flow data are used as input. Table 1 shows the main characteristics of the lines analyzed. The next subsection shows the results of the theoretical study and the case study on the effects of trip time determination on the average additional travel time per passenger. The effects on the probability of on-time departure at the first stop are also analyzed.

The actual data of these lines is gathered by the TriTapt monitoring tool (Muller and Knoppers 2005), which is used by HTM to monitor and analyze the operations of 


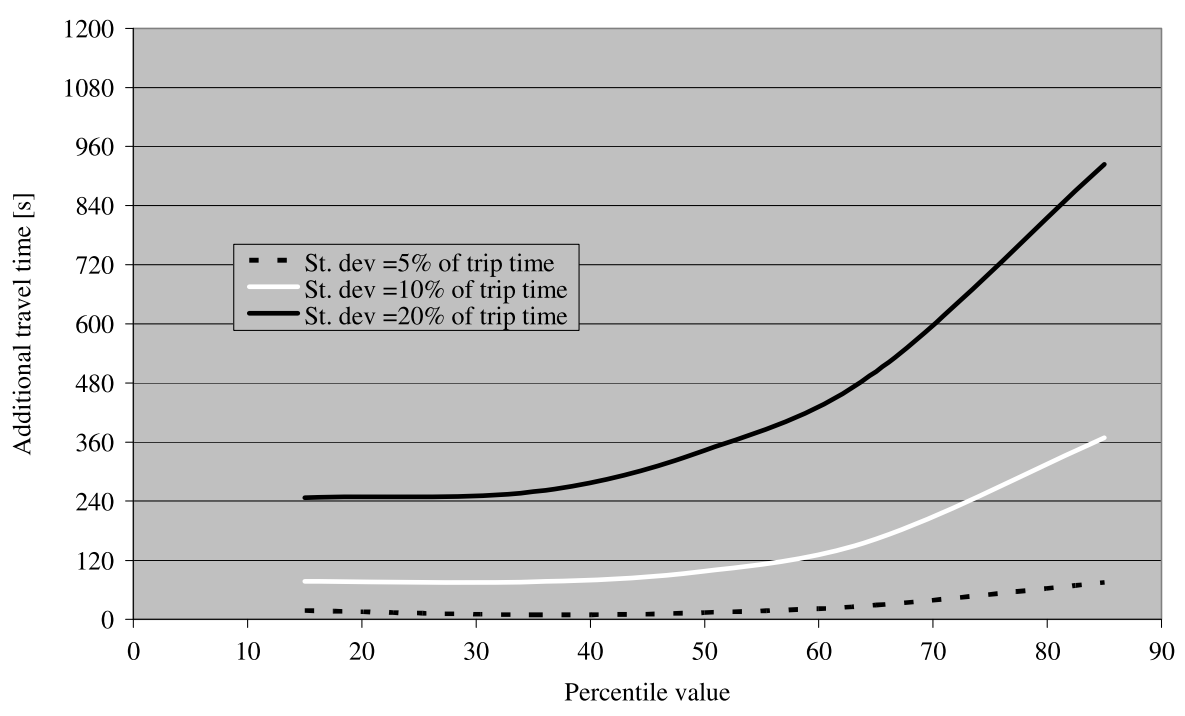

Fig. 6 Average additional travel time per passenger as function of chosen percentile value for a hypothetical line (trip time $30 \mathrm{~min}$, standard deviation 5, 10 and $20 \%$, headway $15 \mathrm{~min}$ )

all tram and bus lines. The period used is February-April 2007. All trips are operated on working days from 6 p.m. to 8 p.m., offering headways of 15 minutes. Operations were not controlled in any way during this period. Concerning the values of $\tau^{\text {early }}$ and

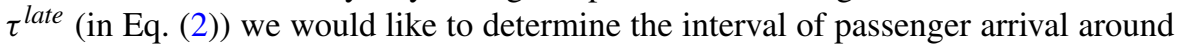
the scheduled vehicle departure time. Van Oort (2011) presents research indicating how early passengers in The Hague arrive at the stop by showing the proportion of passengers arriving a certain amount of minutes before scheduled departure. In that case, they knew or checked the schedule before going to the stop. It appears that about $70 \%$ arrive within 2 minutes before the scheduled departure time. For our analysis, we propose to use a value of 2 minutes for $\tau^{\text {early }}$. This value represents the distribution of the arrival pattern of all passengers (consisting of arrival times of more and less than 2 minutes early) in a proper way.

The value of $\tau^{\text {late }}$ is set to 1 , because it is considered possible that passengers may speed up a little while approaching the stop in combination with drivers waiting for arriving passengers.

\subsection{Trip time determination}

The next section shows the results of an analysis of a hypothetical line and a case study on the effect of trip time determination on average additional travel time per passenger. The effect on the probability of on-time departure at the first stop is also analyzed.

\subsubsection{Analysis of hypothetical line}

The results of the theoretical case are shown in Fig. 6. For three different scheduled trip times, the additional travel time per passenger is shown as a function of 


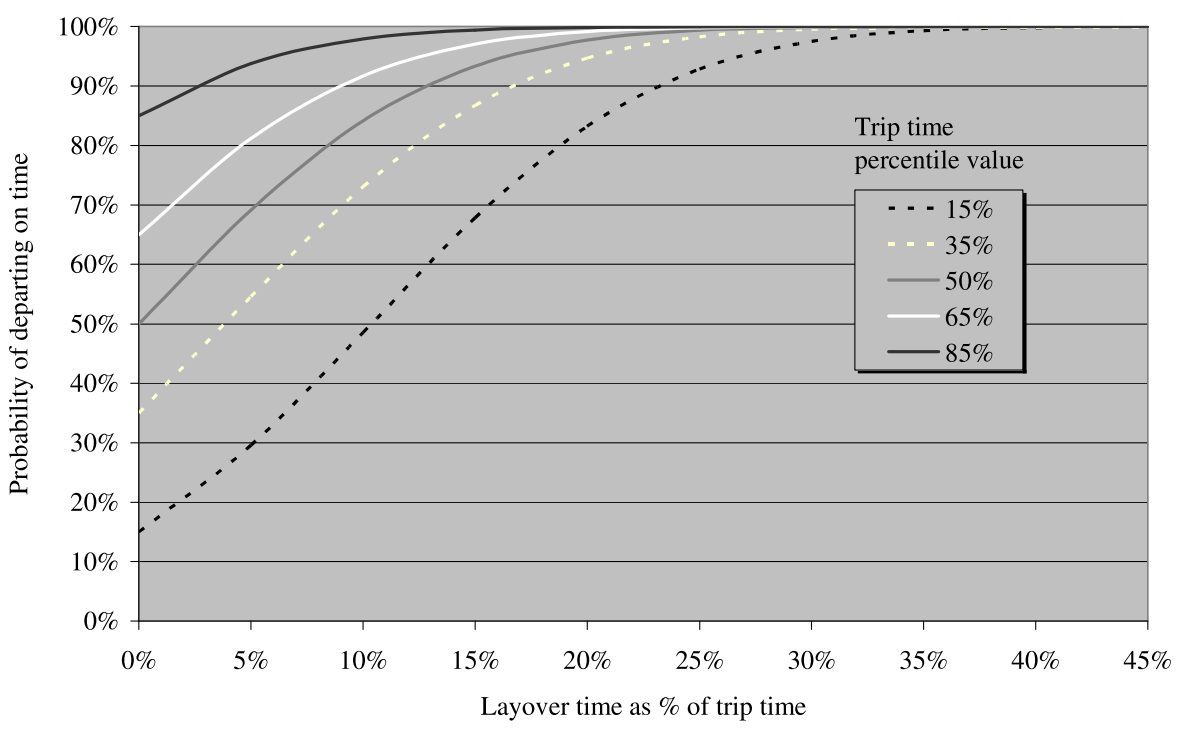

Fig. 7 Probability of on-time departure as a function of percentile value and layover time, hypothetical line

chosen percentile value. This figure illustrates that the average additional travel time increases if the distribution of trip times increases.

The minimum additional travel time is achieved if a 35 percentile (or lower) value is chosen. Depending on the standard deviation of the trip time, the value of additional trip time is between 0.5 and 4 minutes. The difference between the 85 and 35 percentile value lies between 0.5 and 11 minutes. Although a low percentile value will lead to more late vehicles, the prevention of many vehicles driving ahead of schedule will on average decrease the average additional travel time per passenger.

Figure 7 shows the effect of the chosen percentile value on the probability of ontime departure at the first stop, using the scenario for the hypothetical line with a scheduled trip time of 30 minutes and a standard deviation of $10 \%$. Both an increasing layover time and percentile value lead to a higher probability. To ensure punctual departure (in more than $99 \%$ of the trips), a layover time between $15 \%$ and $35 \%$ of the trip time is necessary.

If the percentile value decreases, more layover time is needed to ensure punctual departures in the opposite direction. Nevertheless, this additional time is saved in the trip time, using a lower percentile value. In fact, this is a redistribution of time between driving and layover time, not adjusting total cycle time at all.

\subsubsection{Case study: tram lines in The Hague}

In addition to the analysis of a hypothetical line, a case study is conducted in order to assess the impact of the percentile value choice in practice.

Figure 8 shows the additional travel time for passengers as a function of the chosen percentile value for four tram lines. The figure shows a large increase in additional travel time if the percentile value exceeds $50 \%$. The minimum value, $0.5-1.5 \mathrm{~min}$ - 


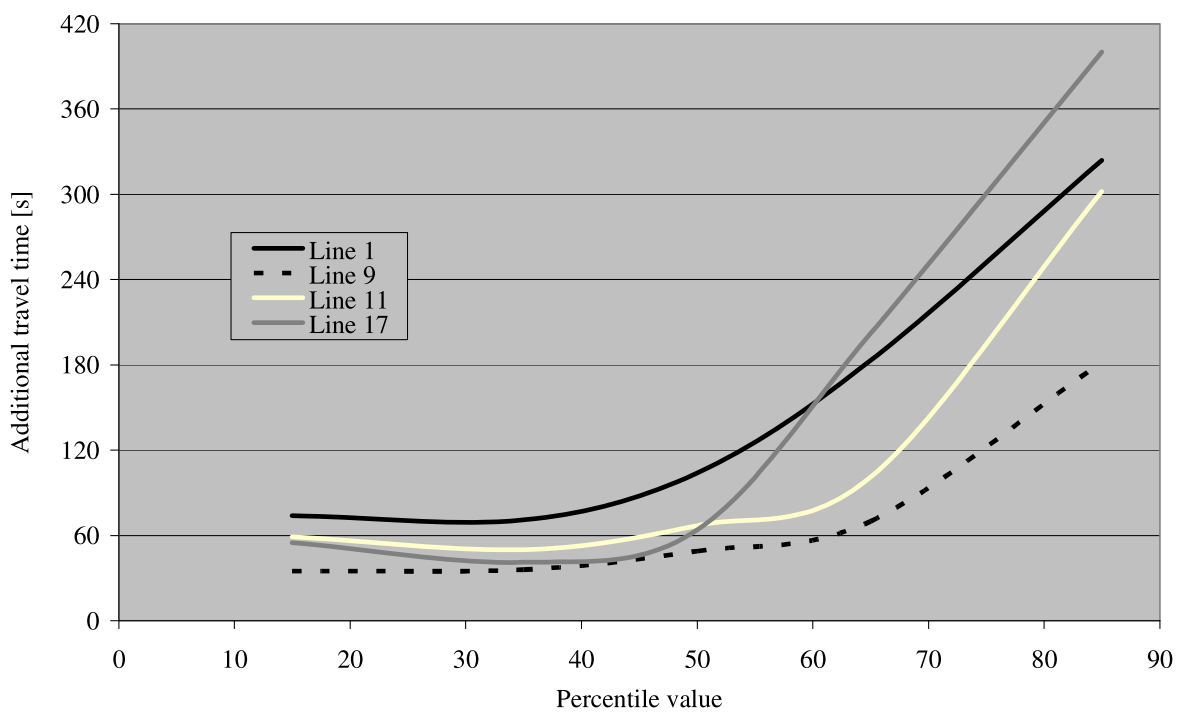

Fig. 8 Average additional travel time per passenger as a function of chosen percentile value

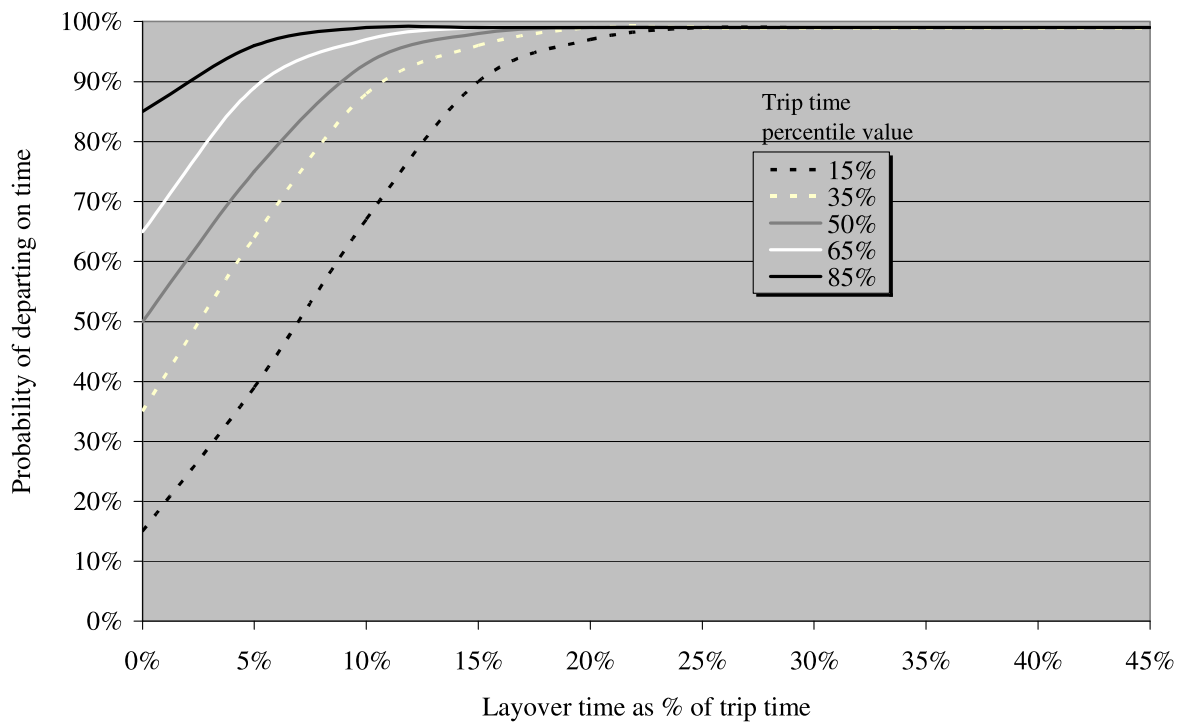

Fig. 9 Probability of on-time departure as a function of percentile value and layover time for tram line 11

utes, is reached when the 35 percentile value is used. This matches the analysis of the hypothetical line. Also shown is a large difference depending on the chosen percentile value. On average, passengers will experience 2 to 5 minutes of extra additional travel time if the 85 percentile value is used, instead of the 35 percentile.

Figure 9 shows the probability of on-time departure from the first stop of tram line 11 as a function of percentile value and layover time. The characteristics of this line 


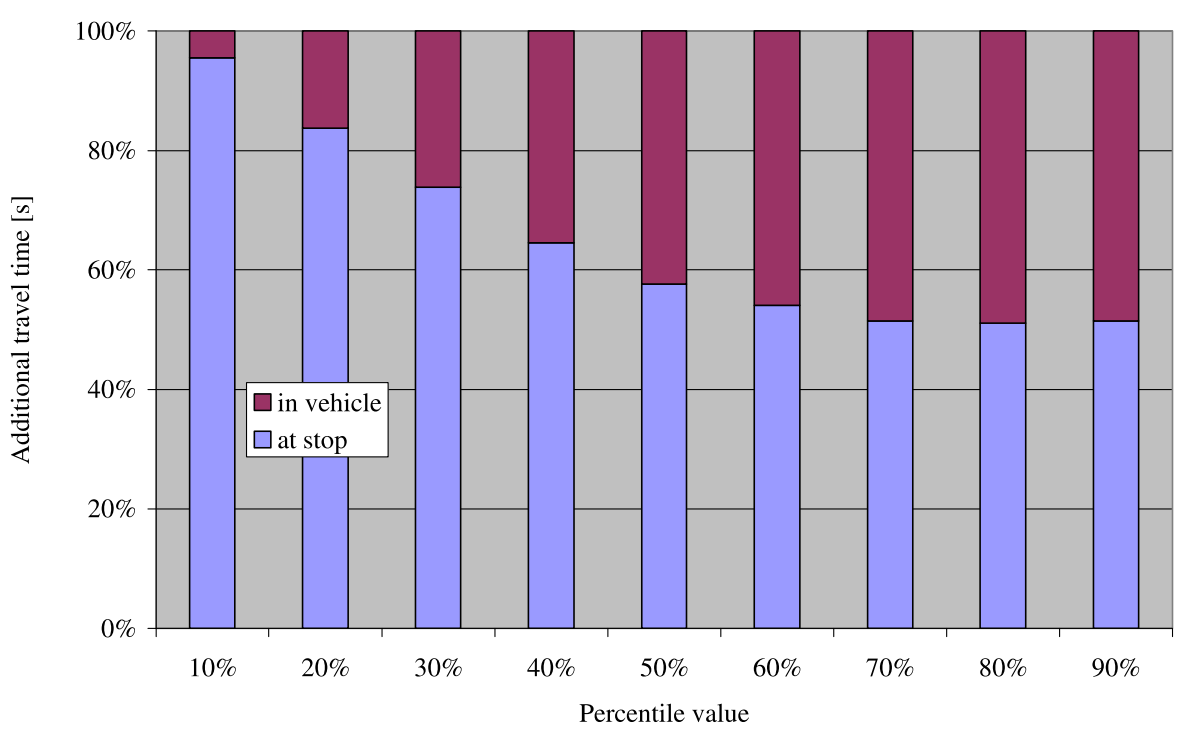

Fig. 10 Average additional travel time per passenger on tram line 1 with four holding points divided into two parts being in the vehicle and at the stop as a function of percentile value

are shown in Table 1. Again, the results match those of the analysis of the hypothetical line. This also confirms that a redistribution of total journey time is necessary: reducing the run time and increasing the layover time. This ensures that passenger travel time is minimized and departure punctuality for the first stop of the next run is not affected.

\subsection{Holding points}

This study also examined the effect of introducing holding points. The former section showed that driving early implies much additional travel time per passenger. In addition to low percentile values, preventing vehicles to operate ahead of schedule may also result in less additional travel time. In the case of holding, the additional travel time consists of two parts: time at the stop and time spent in the vehicle. Figure 10 shows that when using higher percentile values, the additional waiting time shifts from travellers at the stop to travellers in the vehicle. The following sections discuss the effects of the number and location of holding points and the holding point percentile value, which is chosen to set the departure time at the holding point. For each line proper locations for the holding points were chosen, using the considerations given in Sect. 2.2. The following section elaborates on the location of the holding points

\subsubsection{Analysis of hypothetical line}

Figure 11 shows the effect of the number of holding points (0-4) on a hypothetical line with a standard deviation of 4 minutes. It appears that introducing more than two holding points does not significantly reduce additional travel time. However, when 


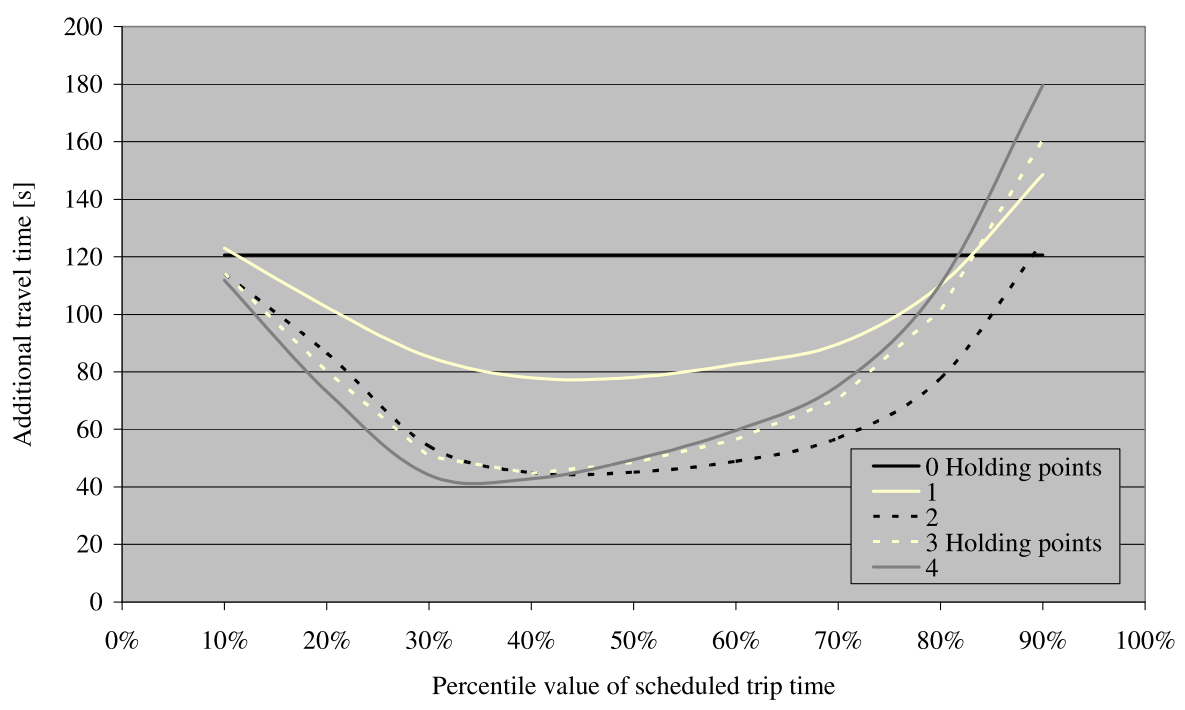

Fig. 11 Average additional travel time per passenger hypothetical line (st. dev. $=4 \mathrm{~min}$ ) as a function of percentile value and number of holding points

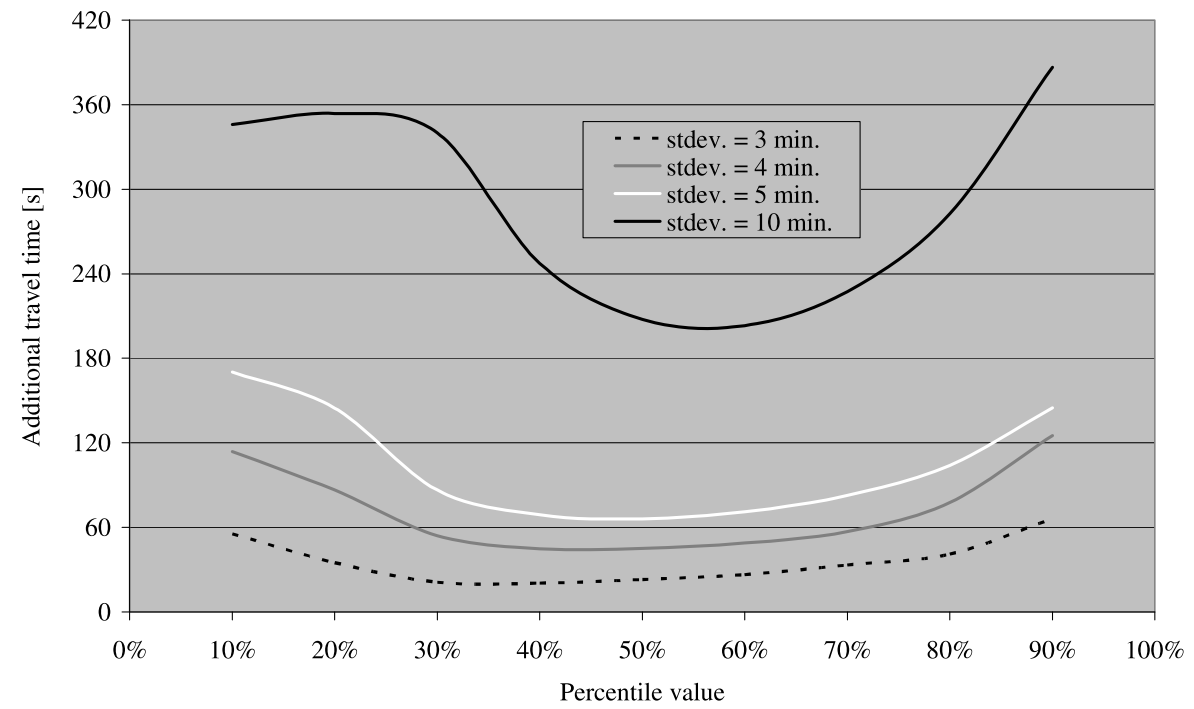

Fig. 12 Average additional travel time per passenger hypothetical line as a function of percentile value and standard deviation of trip time (2 holding points)

more holding points are used, the reduction of additional travel time will be distributed among more passengers. The optimal percentile value lies between 30 and $50 \%$. Figure 12 also shows the effect of the chosen percentile value with which the trip time to the holding point is designed. Besides the number of holding points, this value depends on the standard deviation of the trip time: the higher this deviation, 


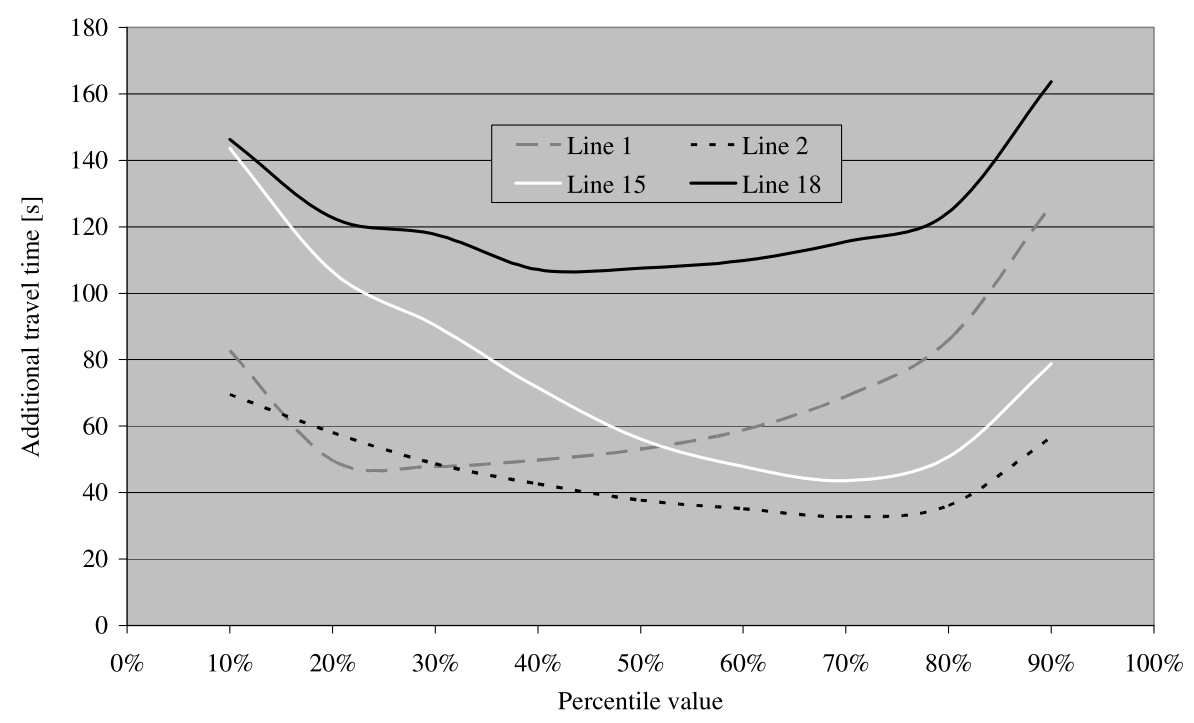

Fig. 13 Average additional travel time per passenger as a function of percentile value ( 2 holding points)

the higher the optimal value of the percentile value. Research of data of real lines supports these conclusions.

\subsubsection{Case study: tram lines in The Hague}

Figure 13 shows the calculated additional travel time by applying two holding points on three tram lines and one bus line. Tram lines 2 and 15 had a small variability of actual trip times. The optimal percentile value is high (60-80-percentile). For tram line 1 the scheduled trip time was short and the realized trip times had a relatively low standard deviation. The optimal percentile value concerning passenger travel time (both in the vehicle and at the stop) is about $20 \%$. Similar to tram line 1 , on bus line 18 the scheduled trip time was tight, but realized trip times are heavily distributed. The optimal percentile value is about $40 \%$. These numbers show a larger bandwidth of optimal values than our research of the hypothetical line, due to more differences between lines. The main reason is the availability of proper locations for holding points (concerning the ratio of through passengers and passengers downstream).

\subsubsection{Location choice of holding points}

As noted above, it is important to choose a good location for a holding point. Figure 14 shows the additional travel time by scheduling a holding point at the beginning (at $1 / 4$ of the length), middle and end (at $3 / 4$ of the length) of the tram line 1 . In general, a holding point in the first part of a line is desirable concerning passengers that may profit (there will still be many boardings ahead) but not yet necessary (the line is not yet greatly deviated). A holding point at the end of a line is necessary because of the higher standard deviation, although only few travellers will benefit. The best location for a holding point thus depends on the distribution of travellers on a line. The case 


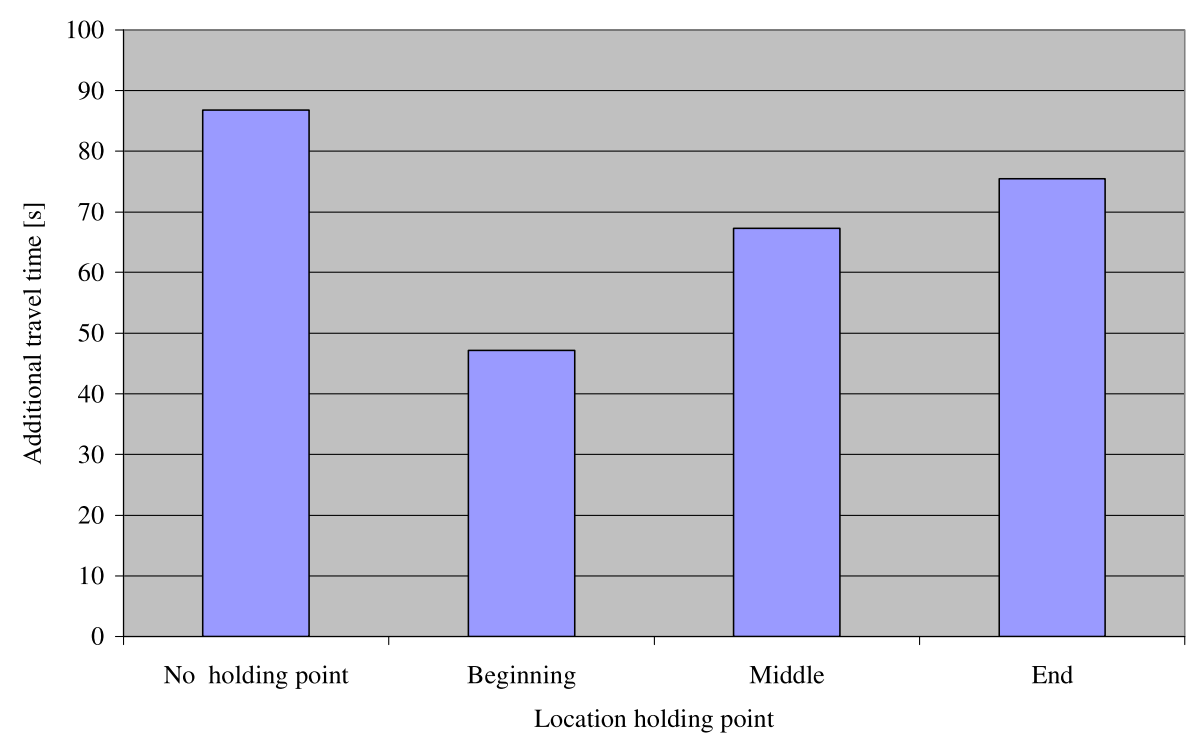

Fig. 14 Average additional travel time per passenger by using one holding point at the beginning, middle and end of tram line 1

of line 1 shows that a holding point at the beginning of that line (at $1 / 4$ of the length) is the optimal location.

\section{Conclusions}

This paper presented research on optimizing the service reliability of urban public transport. Ways of improving reliability by adjusting the timetable are analysed. An analysis of a hypothetical line and a case study show the effect of design choices of timetabling on service reliability of long-headway services. Analysis of actual data shows that the total travel time of passengers is minimized if the 35-percentile value is used to determine the trip time based on historical data. This saves up to $75 \%$ of the additional travel time due to unreliability for all passengers on a line.

If holding points are used (and departing ahead of schedule is not allowed), the additional travel time can be reduced even more. The optimal percentile value when using holding points depends on the following variables:

- The value of the relative standard deviation. The higher the standard deviation, the higher the optimal percentile value.

- Too long or too short timetable trip times. The tighter the schedule (i.e. applying smaller percentile values), the lower the optimal percentile value.

- The number of holding points. The impact of two holding points on the additional travel time is greater than when only one is used, but almost equal to three or four.

- The best location for a holding point depends on the distribution of travellers on a line. 
An analysis of a hypothetical line and a practical study show that designing two holding points, using a 30-60 percentile value minimizes travel time (additional travel times are further reduced up to $60 \%$ ) in terms of both the waiting time at the stops and in the vehicle.

Open Access This article is distributed under the terms of the Creative Commons Attribution License which permits any use, distribution, and reproduction in any medium, provided the original author(s) and the source are credited.

\section{References}

Abkowitz M, Josef R, Tozzi J, Driscoll MK (1987) Operational feasibility of timed transfer in transit systems. J Transp Eng 113:168-177

Carey M (1998) Optimizing scheduled activity times, allowing for behavioural response. Transp Res B 32(5):329-342

Cham LC, Wilson NHM (2006) Understanding bus service reliability, a practical framework using AVL/APC data, Washington, DC

Chang J, Collura J, Dion F, Rakha H (2003) Evaluation of service reliability impacts of traffic signal priority strategies for bus transit. In: Transportation research record no 1841, Washington, DC, pp 23-31

Chowdhury S, Chien S (2001) Dynamic vehicle dispatching at intermodal transfer station. In: Transportation research board 80th annual meeting, Washington, DC

Furth PG, Muller ThHJ (2009) Optimality conditions for public transport schedules with timepoint holding. Public Transp 1(2):87-102 doi:10.1007/s12469-008-0002-5

Hansen IA (1999) Report VK4810: transport operation and management. TU Delft

Levinson HS (1991) Supervision strategies for improved reliability of bus routes, synthesis of transit practice 15, National Cooperative Transit Research and Development Program. Transportation Research Board, Washington, DC

Muller ThHJ, Furth PG (2000) Conditional bus priority at signalized intersections: better service with less traffic disruption. In: Transportation research record no 1731, Washington, DC, pp 23-30

Muller ThHJ, Knoppers P (2004) Modal shift: the objective of improving transit quality. The contribution of off-line trip information to planning, operational control, process management and service information, TU Delft

Muller ThHJ, Knoppers P (2005) TRIp time analysis in public transport. http://tritapt.nl/

Strathman J, Kimple T, Dueker K, Gerhart R, Callas S (2002) Evaluation of transit operations: data applications of Tri-Met's automated bus dispatching system. Transportation 29:321-345

Van Oort N (2009) International benchmark, reliability urban public transport, summary results. Report HTM and Delft University of Technology

Van Oort N (2011) Service reliability and urban public transport design. PhD thesis, Delft University of Technology

Van Oort N, van Nes R (2008) Improving reliability in urban public transport in strategic and tactical design. In: Compendium of papers TRB 87th annual meeting, Washington, DC, pp 1-17

Van Oort N, van Nes R (2009a) Control of public transport operations to improve reliability: theory and practice. In: Transportation research record, No 2112, pp 70-76

Van Oort N, van Nes R (2009b) Regularity analysis for optimizing urban transit network design. Public Transp 1(2): $155-168$

Van Oort N, Wilson NHM, van Nes R (2010) Reliability improvement in short headway transit services: schedule-based and headway-based holding strategies. In: Transportation research record, No 2143, pp 67-76

Wilson NHM, Macchi RA, Fellows RE, Deckoff AA (1992) Improving service on the MBTA green line through better operations control. In: Transportation research record 1361, TRB, National Research Council, Washington, DC, pp 296-304 